\title{
A Novel Prey-Predator Quadratic Harvesting Model via Optimal Control Theory and Hopf Bifurcation
}

\author{
Prabir Panja \& Dipak Kumar Jana \\ Department of Applied Science, Haldia Institute of Technology, Haldia \\ Purba Midnapur-721657, West Bengal, India \\ E-mail: prabirpanja@gmail.com,dipakjana@gmail.com \\ Corresponding Author: Prabir Panja
}

\begin{abstract}
In this investigation, a predator-prey interaction model among Phytoplankton, Zooplankton and Fish has been developed. In the absence of Zooplankton and Fish, it is assumed that Phytoplankton grows logistically. It is assumed that Zooplankton consumes Phytoplankton and Fish consumes Phytoplankton as well as Zooplankton. Holling type I \& II functional responses have been considered to formulate the our proposed model. It is considered that Phytoplankton releases some toxin in the aquatic environment which makes some death in Zooplankton population. Quadratic harvesting is considered on Fish species. Boundedness of the solution of our proposed model has also been studied. Local stability of the system around each equilibrium point has been investigated. Also, the global stability of the interior equilibrium point has been studied. Existence condition of Hopf bifurcation of our proposed system has been studied. It is found that half saturation constant $(\alpha)$ can change the sys-tem dynamics. It is also found that the harvesting rate of Fish (E) and consumption rate of Zooplankton $\left(\gamma_{1}\right)$ has a significant role in the stability of the system. Again, it is found that the harvesting of Fish species will be increased if the selling price of Fish $(p)$ and the annual discount $\left(\delta_{1}\right)$ of Fish production cost increases. It is also found that the optimal harvesting rate of Fish decreases due to the increase of cost (c) of harvesting of Fish. Finally, some numerical simulation results have been presented to verify our analytical findings.
\end{abstract}

Keywords: Phytoplankton; Zooplankton; Fish; Optimal Control; Hopf bifurcation; Routh- Hurwitz criteria 


\section{Introduction}

The study of prey-predator dynamics becoming the most important research topics for ecologists, scientists and applied mathematician due to its universal existence and importance. Mathematical modelling was started with the work of Volterra [32]. After Volterra, many researchers $[5,13,17,18,19,23,24]$ have studied several mathematical models to understand predator-prey dynamics. Effects of seasonality on predator-prey dynamics has been investigated by Levy et al. [16]. Theoretical and evidence based predator-prey model has been studied by Abrams [2]. Wang and Jiang [33] investigated the chaos control of a delayed predator-prey model with the dormancy of predators. Bifurcation analysis of a time-delay model for prey-predator growth with stage-structure has been studied by Qu and Wei [26].

Mainly two types of plankton are found in the aquatic ecosystem such as Phytoplankton and Zooplankton. It is known that Phytoplankton are the primary producer in a food chain. Also, Phytoplankton makes food in the presence of sunlight with the help of chlorophyll. It is found that Phytoplankton forms bloom [6, 10, 20, 29] in the upper surface of the water. It is also shown that when Phytoplankton bloom take place the number of Phytoplankton sharply increases and decreases at short period of time and then returns its original very low level. Zooplankton are a type of heterotrophic plankton that ranges from microscopic organisms to large species, such as jellyfish. Zooplankton are found within large bodies of water, including oceans and freshwater systems. Zooplankton are drifting ecologically important organisms that are an integral component of the food chain. It is experimentally proved that some species of Phytoplankton produce some toxin $[1,6,11,12,22,28,30]$ that makes some death of Zooplankton species. Zhang and Wang [34] have studied a nutrientPhytoplankton-Zooplankton interaction predator prey model. They have shown that the system is persistent as long as the coexisting equilibrium exists. Shi and $\mathrm{Yu}$ [31] have developed a predator-prey model with one Phytoplankton species and two Zooplankton species including two types of delay. They have proved that the delays have a significant role in the stability of the Phytoplankton-Zooplankton system. Several research articles [7, 9, 15, 35] have been published on Phytoplankton-Zooplankton dynamics.

It is known that Fish provides a good source of high quality food that contains many vitamins and minerals. Fish is consumed by many animals as well as human beings throughout the world. It is observed that Fish consumes Phytoplankton as well as Zooplankton. Many people are depending on the harvesting on Fish. Clark [8] studied the optimal harvesting strategy of a particular species. So, the study of the dynamics of Fish in the presence of Phytoplankton and Zooplankton is very much important research topics. There exists very few research articles $[4,14,21,22,27,36]$ in the literature on Phytoplankton-Zooplankton-Fish 
dynamics. But till now, several unexplored dynamics of Phytoplankton-Zooplankton-Fish to be investigated.

The rest of this paper is organized as follows: mathematical model is formulated in Section 2. Boundedness of all solutions of the proposed system is analyzed in Section 3. The existence of different equilibrium points has been determined in Section 4. Local stability analysis of our proposed system around each of the equilibrium points has been studied in Section 5. The global stability of interior equilibrium has been discussed in Section 6. Hopf bifurcation analysis has been done in Section 7. In Section 8, the Optimal control theory is applied to determine optimal harvesting rate of Fish. Numerical simulation results have been presented in section 9. Finally, in the last section we give the main outcomes of the present work.

\section{Assumptions and Model Formulation}

To study the dynamics of Phytoplankton, Zooplankton and Fish, the following assumptions have been made:

- It is considered that at time $t$, the densities of Phytoplankton, Zooplankton and Fish are $P(t), Z(t)$ and $F(t)$ respectively.

- It is assumed that in the absence of Zooplankton and Fish, Phytoplankton grows logistically. Also, Zooplankton consumes Phytoplankton and Fish consumes Phytoplankton as well as Zooplankton.

- It is experimentally proved that consumption rate of Zooplankton by Fish is greater than the consumption rate of phytoplankton by Fish. For this reason, it is assumed that the consumption of Zooplankton and Phytoplankton by Fish have been followed Holling type I \& II functional response respectively.

- It is assumed that Phytoplankton releases some toxin in the aquatic environment which makes some death of Zooplankton species.

- Optimal harvesting of Fish population has been considered. Also, Pontryagin's maximum principle has been used to determine the optimal harvesting of Fish species.

- The intrinsic growth rate of Phytoplankton and the environmental carrying capacity of Phytoplankton have been considered as $r$ and $k$ respectively. 
- Again, the parameters $\beta, \beta_{1}$ and $d$ be taken as the consumption rate of Phytoplankton, the conservation rate of Phytoplankton and the natural death rate of Zooplankton respectively.

- Also, it is assumed that Phytoplankton produces toxin which makes death of Zooplankton. The parameter $\rho$ denotes the rate of releasing the toxic substances produced by per unit biomass of Phytoplankton.

- The parameters $\gamma, \gamma_{1}, s$ and $s_{1}$ denotes the consumption rate of Phytoplankton, the consumption rate of Zooplankton, conservation rate of Phytoplankton and the conservation rate of Zooplankton respectively.

- Again, $\alpha, \delta, q$ and $E$ be the half saturation constant, the death rate of Fish, catchability coefficients and harvesting rate of Fish.

Keeping the above mentioned assumptions in mind, a three species interaction model of Phytoplankton, Zooplankton and Fish has been developed as follows:

$$
\left.\begin{array}{l}
\frac{d P}{d t}=r P\left(1-\frac{P}{k}\right)-\frac{\beta P Z}{\alpha+P}-\frac{\gamma P F}{\alpha+P} \\
\frac{d Z}{d t}=\frac{\beta_{1} P Z}{\alpha+P}-d Z-\frac{\rho P Z}{\alpha+P}-\gamma_{1} Z F \\
\frac{d F}{d t}=\frac{s P F}{\alpha+P}+s_{1} Z F-\delta F-q E F^{2}
\end{array}\right\}
$$

which satisfies the initial conditions $P(0) \geq 0, Z(0) \geq 0$ and $F(0) \geq 0$.

\section{Boundedness of Solutions}

In this section, Boundedness of all solutions of the system (1) has been investigated.

Theorem 1. Solutions of system (1) are bounded.

Proof. Let us define a function $W(t)$, as follows:

$$
W(t)=P(t)+Z(t)+F(t)
$$

Differentiating $W(t)$ with respect to time $t$, it is obtained that

$$
\begin{aligned}
\frac{d W(t)}{d t} & =\frac{d P(t)}{d t}+\frac{d Z(t)}{d t}+\frac{d F(t)}{d t} \\
& =r P\left(1-\frac{P}{k}\right)-\frac{\beta P Z}{\alpha+P}-\frac{\gamma P F}{\alpha+P}+\frac{\beta_{1} P Z}{\alpha+P}-d Z-\frac{\rho P Z}{\alpha+P}-\gamma_{1} Z F \\
& +\frac{s P F}{\alpha+P}+s_{1} Z F-\delta F-q E F^{2} \\
& =r P\left(1-\frac{P}{k}\right)-\frac{P Z}{\alpha+P}\left(\beta-\beta_{1}+\rho\right)-\frac{P F}{\alpha+P}(\gamma-s) \\
& -Z F\left(\gamma_{1}-s_{1}\right)-\delta F-q E F^{2}
\end{aligned}
$$


Now we introduce a positive constant $\sigma$, then the above equation reduced as follows

$$
\begin{aligned}
\frac{d W(t)}{d t}+\sigma W= & r P\left(1-\frac{P}{k}\right)-\frac{P Z}{\alpha+P}\left(\beta-\beta_{1}+\rho\right)-\frac{P F}{\alpha+P}(\gamma-s) \\
- & Z F\left(\gamma_{1}-s_{1}\right)-\delta F-q E F^{2}+\sigma P(t)+\sigma Z(t)+\sigma F(t) \\
\leq & (r+\sigma) P-\frac{r P^{2}}{k}-(d-\sigma) Z-(\delta-\sigma) F-q E F^{2} \\
& \quad \text { Since } \beta>\beta_{1}-\rho, \gamma>s, \gamma_{1}>s_{1} \\
\leq & (r+\sigma) P-\frac{r P^{2}}{k}-q E F^{2}, \text { Since } \sigma=\min \{d, \delta\} \\
\leq & (r+\sigma) P-\frac{r P^{2}}{k}=f(P) \text { (Say) }
\end{aligned}
$$

Therefore,

$$
\begin{gathered}
\max f(P)=\frac{k(r+\sigma)^{2}}{4 r}=M(\text { Say }) \\
\text { i.e., } \frac{d W}{d t}+\sigma W \leq \frac{k}{4 r}(r+\sigma)^{2}
\end{gathered}
$$

provided that $\sigma=\min (\delta, d)$.

Using the theory of differential inequality [3] in the above equation, it is obtained that

$$
0<W(P, Z, F)<\frac{M}{\sigma}\left(1-e^{-\sigma t}\right)+W(P(0), Z(0), F(0)) e^{-\sigma t}
$$

where $M=\frac{k(r+\sigma)^{2}}{4 r \sigma}$.

Now taking limit of the above inequality as $t$ tends to $\infty$, it is obtained that

$$
W(P, Z, F) \leq \frac{M}{\sigma}
$$

From the above equation, it is concluded that the solution of the system lies within the region

$$
\Im=\left[(P, Z, F) \varepsilon R_{+}^{3}: W \leq \frac{M}{\sigma}+\epsilon, \text { for any } \epsilon>0\right] .
$$

Hence the proof.

\section{Equilibrium Points}

In this section, different possible equilibrium points have been determined as follows:

- The trivial equilibrium point $E_{0}=(0,0,0)$. 
- The boundary equilibrium point $E_{1}=(k, 0,0)$.

- The planer equilibrium point $E_{2}=(\hat{P}, \hat{Z}, 0)$ where $\hat{P}=\frac{d \alpha}{\beta_{1}-\rho-d}$ and $\hat{Z}=\frac{r \alpha\left(\beta_{1}-\rho\right)\left(k \beta_{1}-k \rho-k d-d \alpha\right)}{k \beta\left(\beta_{1}-\rho-d\right)^{2}}$.

- The another planer equilibrium point $E_{3}=\left(P^{\prime}, 0, F^{\prime}\right)$ where $P^{\prime}=\frac{\alpha\left(\delta+q E F^{\prime}\right)}{s-\delta-q E^{\prime}}$ and $F^{\prime}=$ $\frac{r\left(\alpha+P^{\prime}\right)\left(1-\frac{P^{\prime}}{k}\right)}{\gamma}>0$.

- The positive equilibrium point $E^{*}=\left(P^{*}, Z^{*}, F^{*}\right)$ where $F^{*}=\frac{1}{\gamma}\left[d-\frac{\left(\beta_{1}-\rho\right) P^{*}}{\alpha+P^{*}}\right]$, $Z^{*}=\frac{1}{s_{1}}\left[\delta+\frac{q E d}{\gamma}-\frac{P^{*}}{\alpha+P^{*}}\left\{s+\frac{q E\left(\beta_{1}-\rho\right)}{\gamma}\right\}\right]$ and $P^{*}$ satisfies the equation and $r\left(1-\frac{P^{*}}{k}\right)-$ $\frac{\beta Z^{*}}{\alpha+P^{*}}-\frac{\gamma F^{*}}{\alpha+P^{*}}=0$.

\section{$5 \quad$ Local Stability Analysis}

In this section, local stability of the proposed system (1) around the equilibrium points has been investigated. The stability of the equilibrium state is determined by the nature of the eigenvalues of the variational matrix $J(P, Z, F)$ is given by

$J(P, Z, F)=\left(\begin{array}{ccc}r-\frac{2 r P}{k}-\frac{\alpha \beta Z}{(\alpha+P)^{2}}-\frac{\alpha \gamma F}{(\alpha+P)^{2}} & \frac{-\beta P}{\alpha+P} & -\frac{\gamma P}{\alpha+P} \\ \frac{\alpha \beta_{1} Z}{(\alpha+P)^{2}}-\frac{\alpha \rho Z}{(\alpha+P)^{2}} & \frac{\beta_{1} P}{\alpha+P}-d-\frac{\rho P}{\alpha+P}-\gamma_{1} F & -\gamma_{1} Z \\ \frac{\alpha s F}{(\alpha+P)^{2}} & s_{1} F & \frac{s P}{\alpha+P}+s_{1} Z-\delta-2 q E F\end{array}\right)$

Theorem 2. The trivial equilibrium point $E_{0}$ is always unstable.

Proof. The variational matrix of the system (1) at $E_{0}$ is given by

$$
J_{E_{0}}=\left(\begin{array}{ccc}
r & 0 & 0 \\
0 & -d & 0 \\
0 & 0 & -\delta
\end{array}\right)
$$

It has eigenvalues $\lambda_{1}=r, \lambda_{2}=-d$ and $\lambda_{3}=-\delta$. It is seen that one eigenvalue $r>0$ is positive. So, we can say that the trivial equilibrium point is always unstable.

Hence the proof.

Theorem 3. The boundary equilibrium point $E_{1}$ is locally asymptotically stable if $k<$ $\max \left\{\frac{\alpha d}{\beta_{1}-\rho-d}, \frac{\delta \alpha}{s-\delta}\right\}$.

Proof. The variational matrix of the system (1) at $E_{1}$ is given by

$$
J_{E_{1}}=\left(\begin{array}{ccc}
-r & -\frac{\beta k}{\alpha+k} & -\frac{\gamma}{\alpha+k} \\
0 & \frac{\beta_{1} k}{\alpha+k}-d-\frac{\rho k}{\alpha+k} & 0 \\
0 & 0 & \frac{s k}{\alpha+k}-\delta
\end{array}\right)
$$


The eigenvalues of the above variational matrix are $\lambda_{1}=-r, \lambda_{2}=\frac{\beta_{1} k}{\alpha+k}-d-\frac{\rho k}{\alpha+k}$ and $\lambda_{3}=\frac{s k}{\alpha+k}-\delta$. According to the condition of stability of a dynamical system, it is known to all that the equilibrium point $E_{1}$ is locally asymptotically stable whenever all the eigenvalues $\lambda_{1}, \lambda_{2}$ and $\lambda_{3}$ must be less than zero. So for the stability of $E_{1}, \lambda_{2}<0$ and $\lambda_{3}<0$ must hold

$$
\begin{aligned}
& \text { i.e., } \frac{\beta_{1} k}{\alpha+k}-d-\frac{\rho k}{\alpha+k}<0 \text { and } \frac{s k}{\alpha+k}-\delta<0 \\
& \text { i.e., } \frac{k\left(\beta_{1}-\rho-d\right)-d \alpha}{\alpha+k}<0 \text { and } \frac{k s-\delta(k+\alpha)}{\alpha+k}<0 \\
& \text { i.e., } k<\left\{\frac{\alpha d}{\beta_{1}-\rho-d}\right\} \text { and } k<\left\{\frac{\delta \alpha}{s-\delta}\right\} \\
& \text { i.e., } k<\max \left\{\frac{\alpha d}{\beta_{1}-\rho-d}, \frac{\delta \alpha}{s-\delta}\right\} .
\end{aligned}
$$

Hence the proof.

Theorem 4. The planer equilibrium point $E_{2}$ is locally asymptotically stable if $B_{1}, B_{3}>0$ and $B_{1} B_{2}>B_{3}$ and otherwise it is unstable.

Proof. The variational matrix of system (1) at $E_{2}$ is given by

$$
J_{E_{2}}=\left(\begin{array}{ccc}
b_{1} & -b_{2} & -b_{3} \\
b_{4} & b_{5} & -b_{6} \\
0 & 0 & b_{7}
\end{array}\right)
$$

where $b_{1}=r-\frac{2 r \hat{P}}{k}-\frac{\alpha \beta \hat{Z}}{(\alpha+\hat{P})^{2}}, b_{2}=\frac{\beta \hat{P}}{\alpha+\hat{P}}, b_{3}=\frac{\gamma \hat{P}}{\alpha+\hat{P}}, b_{4}=\frac{\beta_{1} \alpha \hat{Z}}{(\alpha+\hat{P})^{2}}-\frac{\rho \alpha \hat{Z}}{(\alpha+\hat{P})^{2}}, b_{5}=\frac{\beta_{1} \hat{P}}{\alpha+\hat{P}}-d-\frac{\rho \hat{P}}{\alpha+\hat{P}}, b_{6}=$ $\gamma_{1} \hat{Z}$ and $b_{7}=\frac{s \hat{P}}{\alpha+\hat{P}}+s_{1} \hat{Z}-\delta-2 q E F$.

Then the characteristic equation of the above matrix is given by

$$
x^{3}+B_{1} x^{2}+B_{2} x+B_{3}=0
$$

where $B_{1}=-\left(b_{1}+b_{5}+b_{7}\right), B_{2}=b_{1} b_{7}+b_{5} b_{7}+b_{1} b_{5}+b_{2} b_{4}, B_{3}=-b_{1} b_{5} b_{7}-b_{2} b_{4} b_{7}$. Now by Routh-Hurwitz criteria, the equilibrium point $E_{2}$ is locally asymptotically stable i.e., the eigenvalues of the characteristic equation may have negative real parts if $B_{1}, B_{3}>0$ and $B_{1} B_{2}-B_{3}>0$ and unstable otherwise.

Theorem 5. Another planer equilibrium point $E_{3}$ is locally asymptotically stable if $B_{1}^{\prime}, B_{3}^{\prime}>$ 0 and $B_{1}^{\prime} B_{2}^{\prime}-B_{3}^{\prime}>0$ and unstable otherwise.

Proof. The variational matrix of the system (1) at $E_{3}$ is given by

$$
J_{E_{3}}=\left(\begin{array}{ccc}
b_{11} & -b_{12} & -b_{13} \\
0 & b_{22} & 0 \\
b_{31} & b_{32} & b_{33}
\end{array}\right)
$$


where $b_{11}=r-\frac{2 r P^{\prime}}{k}-\frac{\gamma \alpha F^{\prime}}{\left(\alpha+P^{\prime}\right)^{2}}, b_{12}=\frac{\beta P^{\prime}}{\alpha+P^{\prime}}, b_{13}=\frac{\gamma P^{\prime}}{\alpha+P^{\prime}}, b_{22}=\frac{\beta_{1} P^{\prime}}{\alpha+P^{\prime}}-d-\frac{\rho P^{\prime}}{\alpha+P^{\prime}}-\gamma_{1} F^{\prime}, b_{31}=$ $\frac{s \alpha F^{\prime}}{\left(\alpha+P^{\prime}\right)^{2}}, b_{32}=s_{1} F^{\prime}$ and $b_{33}=\frac{s P^{\prime}}{\alpha+P^{\prime}}-\delta-2 q E F^{\prime}$.

The characteristic equation of the above matrix is given by

$$
y^{3}+B_{1}^{\prime} y^{2}+B_{2}^{\prime} y+B_{3}^{\prime}=0
$$

where $B_{1}^{\prime}=-\left(b_{11}+b_{22}+b_{33}\right), B_{2}^{\prime}=b_{11} b_{33}+b_{22} b_{33}+b_{11} b_{22}+b_{13} b_{31}$ and $B_{3}^{\prime}=-b_{11} b_{22} b_{33}-$ $b_{13} b_{31} b_{22}$. According to Routh-Hurwith criteria, the system will be locally asymptotically stable i.e., the eigenvalues may be negative real parts if $B_{1}^{\prime}, B_{3}^{\prime}>0$ and $B_{1}^{\prime} B_{2}^{\prime}-B_{3}^{\prime}>0$. Otherwise the system becomes unstable.

Theorem 6. The interior equilibrium point $E^{*}$ is locally asymptotically stable if $\sigma_{1}, \sigma_{3}>0$ and $\sigma_{1} \sigma_{2}>\sigma_{3}$ and otherwise it is unstable.

Proof. The variational matrix of system (1) at $E^{*}$ is given by

$$
J_{E^{*}}=\left(\begin{array}{ccc}
\sigma_{11} & -\sigma_{12} & -\sigma_{13} \\
\sigma_{21} & \sigma_{22} & -\sigma_{23} \\
\sigma_{31} & \sigma_{32} & \sigma_{33}
\end{array}\right)
$$

where $\sigma_{11}=r-\frac{2 r P^{*}}{K}-\frac{\alpha \beta Z^{*}}{\left(\alpha+P^{*}\right)^{2}}-\frac{\gamma \alpha F^{*}}{\left(\alpha+P^{*}\right)^{2}}, \sigma_{12}=\frac{\beta P^{*}}{\left(\alpha+P^{*}\right)^{2}}, \sigma_{13}=\frac{\gamma P^{*}}{\alpha+P^{*}}, \sigma_{21}=\frac{\beta_{1} \alpha Z^{*}}{\left(\alpha+P^{*}\right)^{2}}-$ $\frac{\rho \alpha Z^{*}}{\left(\alpha+P^{*}\right)^{2}}, \sigma_{22}=\frac{\beta_{1} P^{*}}{\left(\alpha+P^{*}\right)}-d-\frac{\rho P^{*}}{\left(\alpha+P^{*}\right)}, \sigma_{23}=\frac{\gamma_{1} Z^{*}}{\alpha+P^{*}}, \sigma_{31}=\frac{S \alpha F^{*}}{\left(\alpha+P^{*}\right)^{2}}-\frac{S_{1} Z^{*} F^{*}}{\left(\alpha+P^{*}\right)^{2}}, \sigma_{32}=s_{1} F^{*}$ and $\sigma_{33}=s P^{*}+s_{1} Z^{*}-\delta-2 q E F^{*}$.

The characteristic equation of the above variational matrix $J_{E^{*}}$ is given by

$$
x^{3}+\sigma_{1} x^{2}+\sigma_{2} x+\sigma_{3}=0 .
$$

where $\sigma_{1}=-\left(\sigma_{11}+\sigma_{22}+\sigma_{33}\right), \sigma_{2}=\sigma_{22} \sigma_{33}+\sigma_{23} \sigma_{32}+\sigma_{11} \sigma_{22}+\sigma_{11} \sigma_{33}+\sigma_{12} \sigma_{21}+\sigma_{13} \sigma_{31}$ and $\sigma_{3}=-\sigma_{11} \sigma_{22} \sigma_{33}-\sigma_{11} \sigma_{23} \sigma_{32}-\sigma_{12} \sigma_{31} \sigma_{23}-\sigma_{12} \sigma_{21} \sigma_{33}-\sigma_{31} \sigma_{22} \sigma_{13}+\sigma_{13} \sigma_{21} \sigma_{32}$. According to Routh-Hurwith criteria, the interior equilibrium is locally asymptotically stable if $\sigma_{1}, \sigma_{3}>0$ and $\sigma_{1} \sigma_{2}-\sigma_{3}>0$ holds. Otherwise $E^{*}$ is unstable.

\section{Global Stability Analysis}

In this section, the global stability analysis of the proposed system (1) around the equilibrium points $E^{*}\left(P^{*}, Z^{*}, F^{*}\right)$ has been investigated.

Theorem 7. The system (1) is globally asymptotically stable around the interior equilibrium point $E^{*}\left(P^{*}, Z^{*}, F^{*}\right)$ if $\frac{r}{k}>\beta Z^{*}+\gamma F^{*}$ hold. 
Proof. Let us choose a suitable Lyapunov function

$$
V=P-P^{*} \log \left(\frac{P}{P^{*}}\right)+k_{1}\left(Z-Z^{*} \log \frac{Z}{Z^{*}}\right)+k_{2}\left(F-F^{*} \log \frac{F}{F^{*}}\right)
$$

Taking time derivative of the above equation, we have

$$
\frac{d V}{d t}=\frac{d P}{d t}\left(\frac{P-P^{*}}{P}\right)+k_{1}\left(\frac{Z-Z^{*}}{Z}\right) \frac{d Z}{d t}+k_{2}\left(\frac{F-F^{*}}{F}\right) \frac{d F}{d t}
$$

Substituting the value of $\frac{d P}{d t}, \frac{d Z}{d t}$ and $\frac{d F}{d t}$ in the above equation, it is obtained that

$$
\begin{aligned}
\frac{d V}{d t} & =\left(P-P^{*}\right)\left\{r\left(1-\frac{P}{k}\right)-\frac{\beta Z}{\alpha+P}-\frac{\gamma F}{\alpha+P}\right\}+k_{1}\left(Z-Z^{*}\right)\left\{\frac{\beta_{1} P}{\alpha+P}-d-\frac{\rho P}{\alpha+P}-\gamma_{1} F\right\} \\
& +k_{2}\left(F-F^{*}\right)\left\{\frac{s P}{\alpha+P}+s_{1} Z-\delta-q E F\right\} \\
& =\left(P-P^{*}\right)\left\{r\left(1-\frac{P}{k}\right)-\frac{\beta Z}{\alpha+P}-\frac{\gamma F}{\alpha+P}-r\left(1-\frac{P^{*}}{k}\right)+\frac{\beta Z^{*}}{\alpha+P^{*}}+\frac{\gamma F^{*}}{\alpha+P^{*}}\right\} \\
& +k_{1}\left(Z-Z^{*}\right)\left\{\frac{\beta_{1} P}{\alpha+P}-d-\frac{\rho P}{\alpha+P}-\gamma_{1} F-\frac{\beta_{1} P^{*}}{\alpha+P^{*}}+d+\frac{\rho P^{*}}{\alpha+P^{*}}+\gamma_{1} F^{*}\right\} \\
& +k_{2}\left(F-F^{*}\right)\left\{\frac{s P}{\alpha+P}+s_{1} Z-\delta-q E F-\frac{s P^{*}}{\alpha+P^{*}}-s_{1} Z^{*}+\delta+q E F^{*}\right\}
\end{aligned}
$$

After simplification, it is obtained that

$$
\begin{aligned}
\frac{d V}{d t} & \leq\left(-\frac{r}{k}+\beta Z^{*}+\gamma F^{*}\right)\left(P-P^{*}\right)^{2}-k_{2} q E\left(F-F^{*}\right)^{2} \\
& +\left(P-P^{*}\right)\left(Z-Z^{*}\right)\left\{k_{1} \alpha\left(\beta_{1}-\rho\right)-\beta P^{*}-\beta \alpha\right\} \\
& +\left(P-P^{*}\right)\left(F-F^{*}\right)\left\{k_{2} s \alpha-\gamma P^{*}-\gamma \alpha\right\}+\left(Z-Z^{*}\right)\left(F-F^{*}\right)\left\{k_{2} s_{1}-k_{1} \gamma_{1}\right\}
\end{aligned}
$$

If we choose $k_{1}=\frac{\beta\left(\alpha+P^{*}\right)}{\alpha\left(\beta_{1}-\rho\right)}, k_{2}=\frac{\gamma\left(\alpha+P^{*}\right)}{s \alpha}$ and $k_{2} s_{1}=k_{1} \gamma_{1}$, then the above equation reduces the following form

$$
\begin{aligned}
\frac{d V}{d t} & \leq-\left(\frac{r}{k}-\beta Z^{*}-\gamma F^{*}\right)\left(P-P^{*}\right)^{2}-k_{2} q E\left(F-F^{*}\right)^{2} \\
& <0, \text { whenever } \frac{r}{k}>\beta Z^{*}+\gamma F^{*}
\end{aligned}
$$

Hence the proof.

\section{Bifurcation Analysis}

In this section, Hopf bifurcation analysis of system (1) around the interior equilibrium point has been discussed. The main objective of this investigation is to study the impacts of 
change of model parameters on the dynamics of our proposed system. In this paper, we have considered $\alpha$ as a bifurcation parameter and $\alpha^{*}$ represents the critical value or the bifurcating value of the concerned bifurcation parameter.

Theorem 8. The positive equilibrium $E^{*}$ enters into Hopf bifurcation as $\alpha$ varies over $\Re$. Let $\phi:(0, \infty) \rightarrow \Re$ be the following continuously differential function of $\alpha$.

$$
\phi(\alpha)=\sigma_{1}(\alpha) \sigma_{2}(\alpha)-\sigma_{3}(\alpha)
$$

Let $\alpha^{*}$ be a positive root of the equation $\phi(\alpha)=0$. Therefore, the Hopf bifurcation of the interior equilibrium $E\left(P^{*}, Z^{*}, F^{*}\right)$ occurs at $\alpha=\alpha^{*}$ if and only if

(i) $\phi\left(\alpha^{*}\right)=0$

(ii) $L_{2}\left(\alpha^{*}\right) L_{4}\left(\alpha^{*}\right)+L_{1}\left(\alpha^{*}\right) L_{3}\left(\alpha^{*}\right) \neq 0$.

Proof. By the condition $\phi(\alpha)=0$, then the characteristic equation of the variational matrix $V_{E^{*}}$ from Theorem 6. can be written as

$$
\left(x^{2}+\sigma_{2}\right)\left(x+\sigma_{1}\right)=0
$$

Let us considered the roots of the above equation are $\rho_{1}, \rho_{2}$ and $\rho_{3}$. Let the pair of imaginary roots at $\alpha=\alpha^{*}$ are $\rho_{1}, \rho_{2}$, then we have $\rho_{3}=-\sigma_{1}$ and $\left.\rho_{1}, \rho_{2}= \pm i \sqrt{(} \sigma_{2}\right)$.

As $\psi\left(\alpha^{*}\right)$ is a continuous function of all its roots so there exists an open interval $\left(\alpha^{*}-\epsilon, \alpha^{*}+\epsilon\right)$ where $\rho_{1}$ and $\rho_{2}$ are complex conjugate for $\alpha$. Suppose that their general forms in this neighborhood are

$$
\begin{aligned}
& \rho_{1}(\alpha)=\chi(\alpha)+i \xi(\alpha) \\
& \rho_{2}(\alpha)=\chi(\alpha)-i \xi(\alpha)
\end{aligned}
$$

Now we shall verify the transversality condition $\left.\frac{d\left(R e \rho_{j}\right)}{d \alpha}\right|_{\alpha=\alpha^{*}} \neq 0, j=1,2$. Substituting, $\rho_{j}(\alpha)=\chi(\alpha) \pm i \xi(\alpha)$ into the characteristic equation and calculating the derivative, it is obtained that

$$
\begin{aligned}
& L_{1}(\alpha) \chi^{\prime}(\alpha)-L_{2}(\alpha) \xi^{\prime}(\alpha)+L_{3}(\alpha)=0 \\
& L_{2}(\alpha) \chi^{\prime}(\alpha)+L_{1}(\alpha) \xi^{\prime}(\alpha)+L_{4}(\alpha)=0
\end{aligned}
$$

where

$$
\begin{aligned}
& L_{1}(\alpha)=3 \chi^{2}-3 \xi^{2}+2 \sigma_{1} \chi+\sigma_{2} \\
& L_{2}(\alpha)=6 \chi \xi+2 \sigma_{1} \xi \\
& L_{3}(\alpha)=\sigma_{1}^{\prime} \chi^{2}-\sigma_{1}^{\prime} \xi^{2}+\sigma_{2}^{\prime} \chi+\sigma_{3}^{\prime} \\
& L_{4}(\alpha)=2 \sigma_{1}^{\prime} \chi \xi+\sigma_{2}^{\prime} \xi
\end{aligned}
$$


Solving for $\xi^{\prime}(\alpha)$ at $\alpha=\alpha^{*}$, it is obtained that

$$
\left.\frac{d\left(\operatorname{Re} \rho_{j}(\alpha)\right)}{d \alpha}\right|_{\alpha=\alpha^{*}}=\chi^{\prime}\left(\alpha^{*}\right)=-\frac{L_{2}\left(\alpha^{*}\right) L_{4}\left(\alpha^{*}\right)+L_{1}\left(\alpha^{*}\right) L_{3}\left(\alpha^{*}\right)}{L_{1}^{2}\left(\alpha^{*}\right)+L_{2}^{2}\left(\alpha^{*}\right)} \neq 0
$$

if $L_{2}\left(\alpha^{*}\right) L_{4}\left(\alpha^{*}\right)+L_{1}\left(\alpha^{*}\right) L_{3}\left(\alpha^{*}\right) \neq 0$. Thus the transversality condition holds and hence Hopf bifurcation occurs at $\alpha=\alpha^{*}$.

Hence the proof.

\section{Optimal Harvesting}

In this section, the optimal harvesting of Fish species has been determined. It is known to all that the harvesting is an important issue for the economic development of a country. Again, continuous and unscientific harvesting of a Fish species can extinct the species. So, the optimal harvesting is necessary for the conservation of Fish species. The emphasis of this section is on the profit making aspect of Fisheries. It is a thorough study of the optimal harvesting policy and the profit earned by harvesting considering quadratic costs and conservation of Fish species. The prime reason for using quadratic costs is that it allows to derive an analytic expression for the optimal harvesting. It is assumed that the price is a function which decreases with the increasing biomass. Thus to maximize the total discounted net revenues from the Fishery, the optimal control problem can be formulated as follows:

$$
J(E)=\int_{t_{0}}^{t_{f}} e^{-\delta_{1} t}\left[\left(p-\omega q_{1} E F^{2}\right) q_{1} E F^{2}-c E\right] d t
$$

where $\delta_{1}$ is the annual discount rate of cost of Fish production, $p$ is the constant price per unit biomass of Fish, $c$ is the constant cost of the cost of harvesting of Fish species and $\omega$ is the economic constant. The problem (4) can be solved by applying Pontryagin's maximum principle [25] subject to the model system (1) and control constraints $0 \leq E \leq E_{\max }$. The convexity of the objective function with respect to $E$, the linearity of the differential equations in the control and the compactness of the range values of the state variables can be combined to give the existence of the optimal control. Suppose $E_{\delta_{1}}$ is an optimal control with corresponding states $P_{\delta_{1}}, Z_{\delta_{1}}$ and $F_{\delta_{1}}$. We take $A_{\delta_{1}}\left(P_{\delta_{1}}, Z_{\delta_{1}}, F_{\delta_{1}}\right)$ as optimal equilibrium point. Here, we intend to derive optimal control $E_{\delta_{1}}$ such that

$$
J\left(E_{\delta_{1}}\right)=\max \{J(E): E \in U\}
$$

where $U$ is the control set defined by $U=\left\{E:\left[t_{0}, t_{f}\right] \rightarrow\left[0, E_{\text {max }}\right] \mid E\right.$ is Lebesgue measurable $\}$. The Hamiltonian of this optimal control problem is given by

$$
\begin{aligned}
H & =\left\{\left(p-\omega q_{1} E F^{2}\right) q_{1} E F^{2}-c E\right\}+\lambda_{1}\left\{r P\left(1-\frac{P}{k}\right)-\frac{\beta P Z}{\alpha+P}-\frac{\gamma P F}{\alpha+P}\right\} \\
& +\lambda_{2}\left\{\frac{\beta_{1} P Z}{\alpha+P}-d Z-\frac{\rho P Z}{\alpha+P}-\gamma_{1} Z F\right\}+\lambda_{3}\left\{\frac{s P F}{\alpha+P}+s_{1} Z F-\delta F-q E F^{2}\right\}
\end{aligned}
$$


where $\lambda_{i}, i=1,2,3$ are the adjoint variables. The transversality condition for problem gives $\lambda_{i}\left(t_{f}\right)=0, i=1,2,3$.

Now, it is possible to find the characterization of the optimal control $E_{\delta_{1}}$. On the set $t: 0<E_{\delta_{1}}(t)<E_{\max }$, we have

$$
\frac{\partial H}{\partial E}=p q_{1} F-2 \omega q_{1}^{2} F^{4} E-c-q F^{2} \lambda_{3}
$$

Thus at $A_{\delta_{1}}\left(P_{\delta_{1}}, Z_{\delta_{1}}, F_{\delta_{1}}\right), E=E_{\delta_{1}}(t)$ and $\frac{\partial H}{\partial E}=p q_{1} F-2 \omega q_{1}^{2} F^{4} E-c-q F^{2} \lambda_{3}=0$. This implies that

$$
E_{\delta_{1}}=\left\{\frac{p q_{1} F_{\delta_{1}}-c-q F_{\delta_{1}}^{2} \lambda_{3}}{2 \omega q_{1}^{2} F_{\delta_{1}}^{4}}\right\}
$$

Now the adjoint equations at the point $A_{\delta_{1}}\left(P_{\delta_{1}}, Z_{\delta_{1}}, F_{\delta_{1}}\right)$ are

$$
\begin{aligned}
\frac{d \lambda_{1}}{d t} & =\delta_{1} \lambda_{1}-\left(\frac{\partial H}{\partial P}\right)_{A_{\delta_{1}}} \\
& =\delta_{1} \lambda_{1}-\lambda_{1}\left\{r\left(1-\frac{2 P_{\delta_{1}}}{k}\right)-\frac{\beta Z_{\delta_{1}}}{\left(\alpha+P_{\delta_{1}}\right)^{2}}-\frac{\gamma F_{\delta_{1}}}{\left(\alpha+P_{\delta_{1}}\right)^{2}}\right\} \\
& +\lambda_{2}\left\{\frac{\beta_{1} Z_{\delta_{1}}}{\left(\alpha+P_{\delta_{1}}\right)^{2}}-\frac{\rho Z_{\delta_{1}}}{\left(\alpha+P_{\delta_{1}}\right)^{2}}\right\}+\lambda_{3}\left\{\frac{s F_{\delta_{1}}}{\left(\alpha+P_{\delta_{1}}\right)^{2}}\right\} \\
\frac{d \lambda_{2}}{d t} & =\delta_{1} \lambda_{2}-\left(\frac{\partial H}{\partial Z}\right)_{A_{\delta_{1}}} \\
& =\delta_{1} \lambda_{2}+\lambda_{1}\left\{\frac{\beta P_{\delta_{1}}}{\alpha+P_{\delta_{1}}}\right\}-\lambda_{2}\left\{\frac{\beta_{1} P_{\delta_{1}}}{\alpha+P_{\delta_{1}}}-d-\frac{\rho P_{\delta_{1}}}{\alpha+P_{\delta_{1}}}-\gamma_{1} F_{\delta_{1}}\right\}-\lambda_{3}\left\{s_{1} F_{\delta_{1}}\right\} \\
\frac{d \lambda_{3}}{d t} & =\delta_{1} \lambda_{3}-\left(\frac{\partial H}{\partial F}\right)_{A_{\delta_{1}}} \\
& =\delta_{1} \lambda_{3}-\left\{2 p q_{1} F_{\delta_{1}}-4 W q_{1}^{2} E^{2} F_{\delta_{1}}^{4}\right\}+\frac{\lambda_{1} \gamma P_{\delta_{1}}}{\alpha+P_{\delta_{1}}}+\lambda_{2} \gamma_{1} Z_{\delta_{1}} \\
& -\lambda_{3}\left\{\frac{s P_{\delta_{1}}}{\alpha+P_{\delta_{1}}}-s_{1} Z_{\delta_{1}}-\delta-2 q_{1} E F_{\delta_{1}}\right\}
\end{aligned}
$$

The equations (5)-(7) are the first order system of simultaneous differential equations and it is easy to get the analytical solution of the equation with the help of initial conditions $\lambda_{i}\left(t_{f}\right)=0, i=1,2,3$.

\section{$9 \quad$ Numerical Illustrations}

In this section, the dynamical behavior of the proposed model (1) has been investigated numerically using MATLAB software. Due to unavailability of real data, a set of hypothetical 
values of parameters has been considered as $r=2.0, k=120, \beta=0.6, \alpha=4.0, \gamma=0.5, \beta_{1}=$ $0.4, d=0.03654, \rho=0.1, s=0.2, s_{1}=0.2, \delta=0.6, q=0.01, E=0.4, \gamma_{1}=0.1$. Using this set of parametric values, Figure 1 has been drawn. From this figure, it is seen that the interior equilibrium point is locally asymptotically stable. So, we can say that Phytoplankton, Zooplankton and Fish species coexist.

Using this set of parametric values $r=2.0, k=120, \beta=0.6, \gamma=0.5, \beta_{1}=0.9, d=$ $0.3654, \rho=0.2, \gamma_{1}=0.6, s=0.2, s_{1}=0.2, \delta=0.6, q=0.01, E=0.1$, bifurcation diagram of system (1) with respect to $\alpha$ has been drawn in Figure 2. We have numerically calculated the critical value of $\alpha$ as 1.18. From this Figure, it is observed that the system loses it's stability whenever $\alpha<1.18$, but the system continues stable steady state behaviour for $\alpha>1.18$. So, it can be concluded that the half saturation constant has a significant role on the stability of our proposed system.
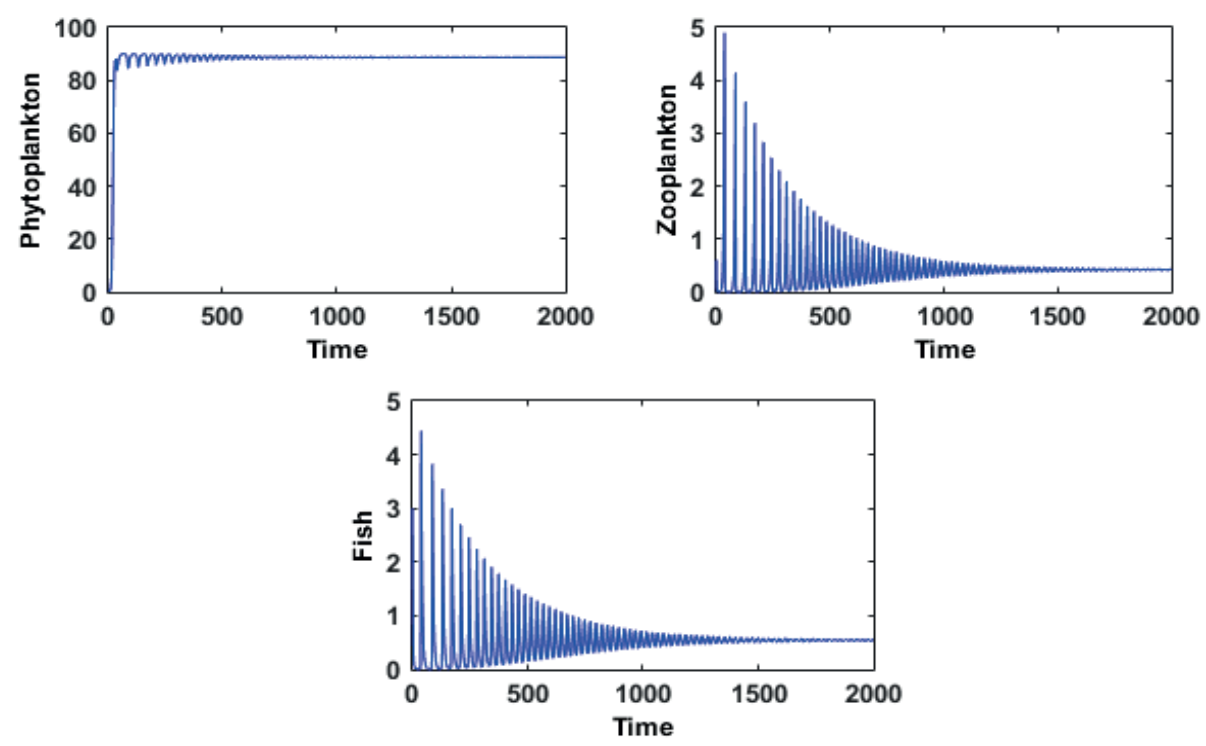

Figure 1: Time evolution for population densities. 

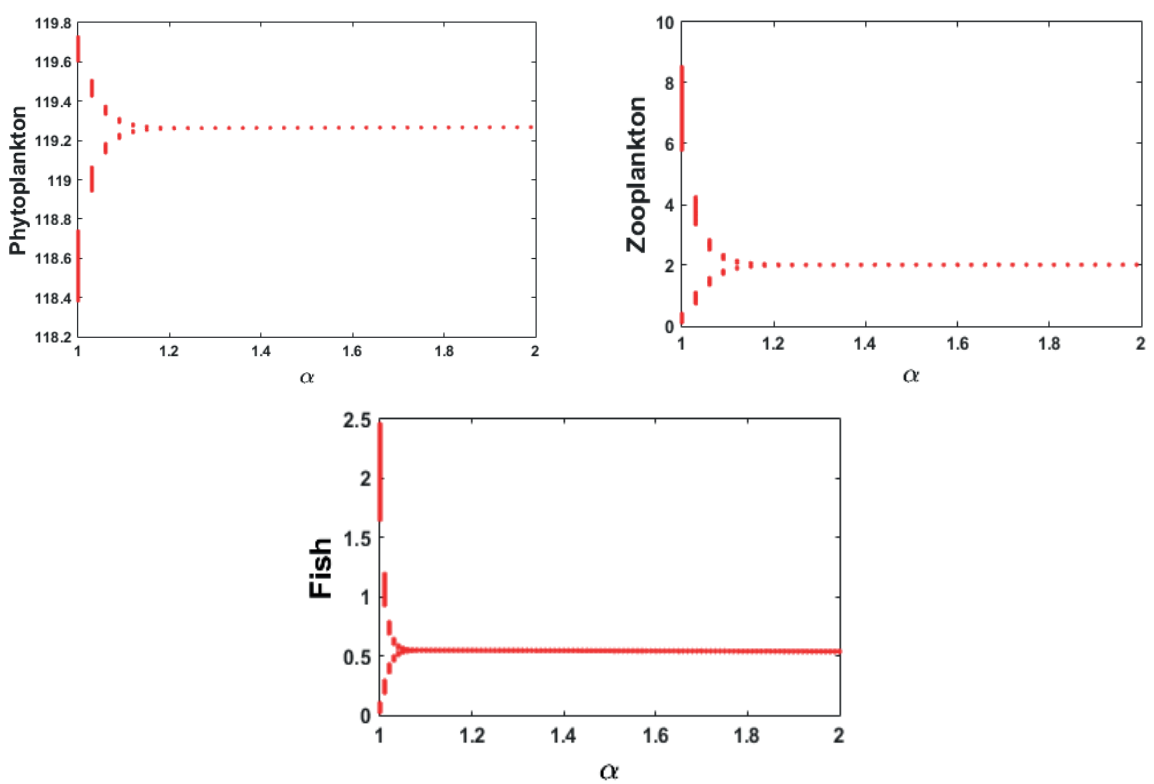

Figure 2: Bifurcation diagrams as a function of half saturation constant $(\alpha)$ with respect to population densities.

Bifurcation diagram of system (1) with respect to $E$ has been presented in Figure 3 for the set of parametric values $r=0.4, k=90, \beta=0.6, \alpha=1.0, \gamma=0.5, \beta_{1}=0.9, d=0.3654, \rho=$ $0.2, \gamma_{1}=0.6, s=0.4, s_{1}=0.5, \delta=0.6, q=0.01$. For this set of parametric values, the critical value of $E$ has been calculated as 0.014. From this figure, it is seen that as the value of $E$ increases, then the system remains unstable within $0.01 \leq E \leq 0.014$, but the system continues stable steady state behaviour for $E>0.014$. It can be concluded that the harvesting of Fish species may be responsible for the stability of the system. 

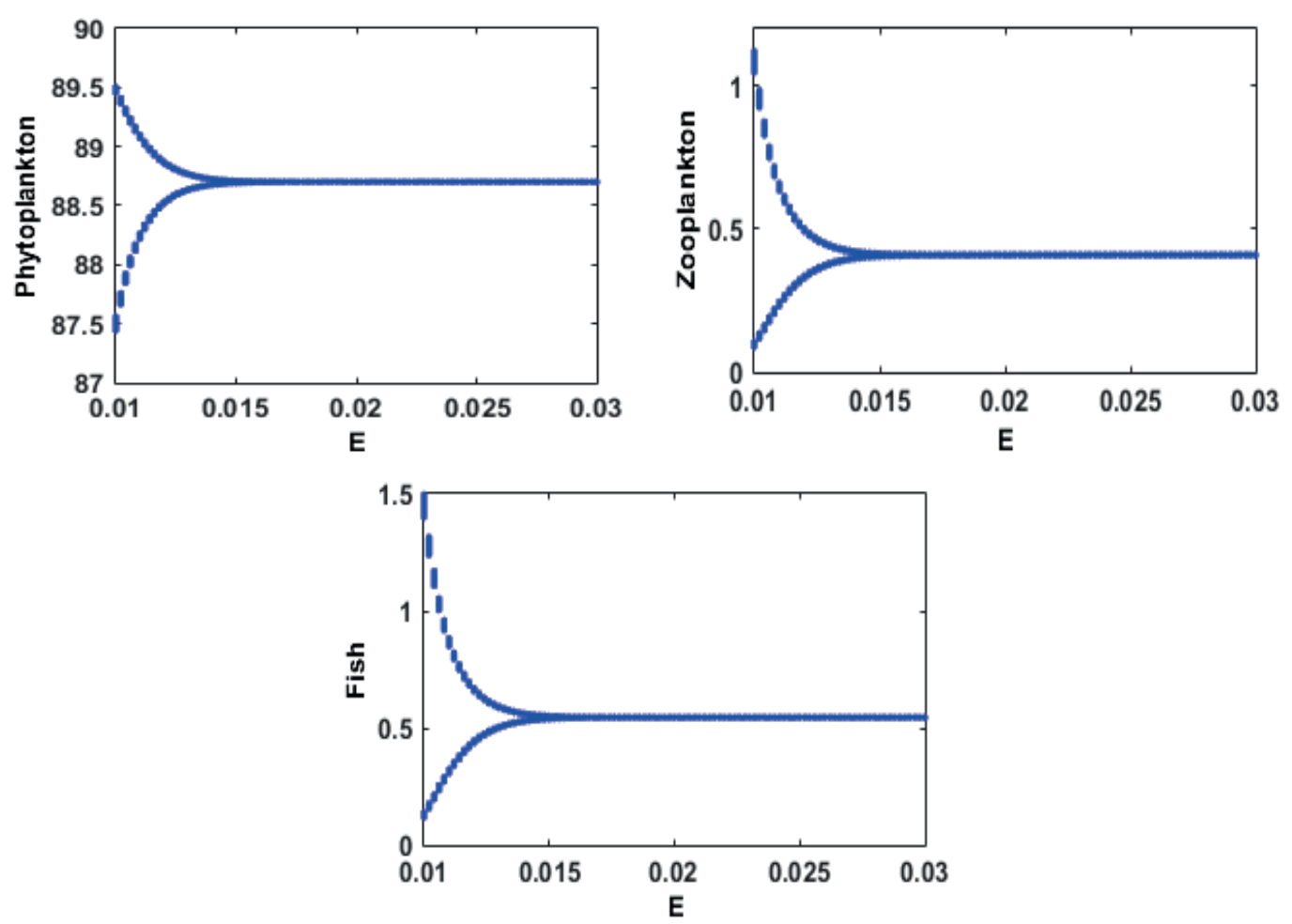

Figure 3: Bifurcation diagrams as a function of harvesting rate of Fish species $(E)$ with respect to population densities.

Using the set of parametric values $r=2.0, k=120, \beta=0.6, \alpha=4.0, \gamma=0.5, \beta_{1}=0.4, d=$ $0.03654, \rho=0.1, s=0.2, s_{1}=0.2, \delta=0.6, q=0.01, E=0.4$, bifurcation diagram of system (1) has been presented in Figure 4. The critical value of $\gamma_{1}$ has been obtained as 0.00604 . From this figure, it is observed that the system remains unstable or continues the oscillatory behaviour for $\gamma_{1}<0.00604$, but the system continues stable steady state behaviour for $\gamma_{1} \geq 0.00604$. So, it can be concluded that due to availability of food for Fish may be stabilized the system. 

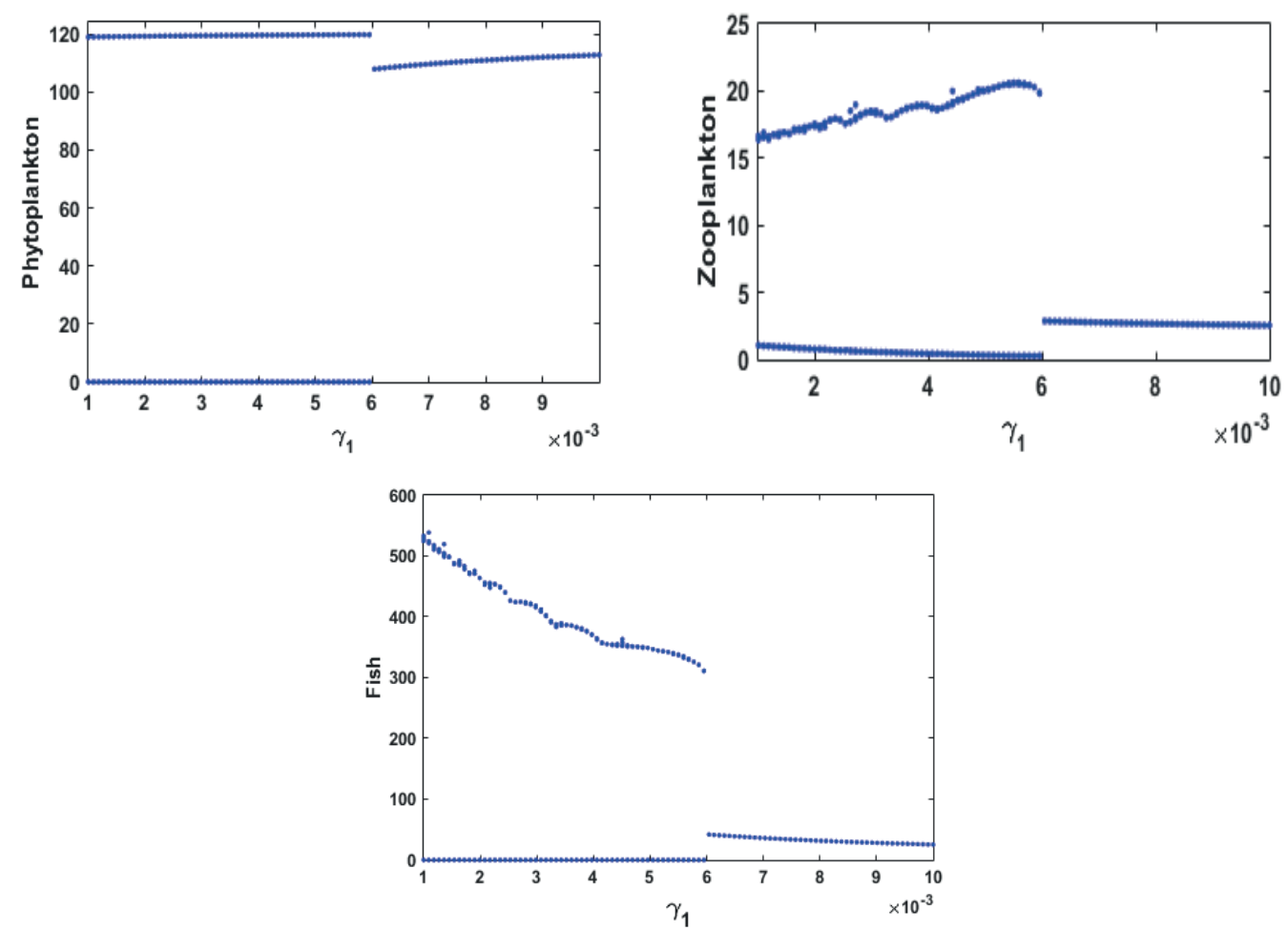

Figure 4: Bifurcation diagrams as a function of consumption rate of Zooplankton $\left(\gamma_{1}\right)$ by Fish species with respect to population densities.

Now the numerical solution of the optimal harvesting problem has been solved by using the set of parametric values: $r=2.0, k=100.0, \beta=0.6, \alpha=1.0, \gamma=0.7, \beta_{1}=0.5, d=$ $0.345, \rho=0.2, \gamma_{1}=0.6, s=0.5, s_{1}=0.4, \delta=0.01, q=0.02$. First we solve the state equations (1) by using Forward Runge-Kutta method within a specified time interval. Next, we solve the co-state equations (5)-(7) by using Backward Runge-Kutta method to solve the optimal harvesting problem (4). Then the optimal harvesting results are shown in the following figures with respect to selling price of Fish $(p)$, the cost of harvesting $(c)$ and instantaneous annual discount rate $\left(\delta_{1}\right)$ respectively. From Figure 5, it is observed that as the selling price of unit biomass of Fish increases, then the optimal harvesting rate of Fish species gradually increases. From Figure 6, it is observed that as the cost of harvesting of Fish increases, then the optimal harvesting of Fish first, gradually decreases after that it goes to the equilibrium level. From Figure 7, it is seen that as the annual discount rate of selling price increases, then the optimal harvesting of Fish gradually increases. So, it can be concluded that the increase of annual discount rate of selling price can increase the optimal harvesting rate of Fish. 


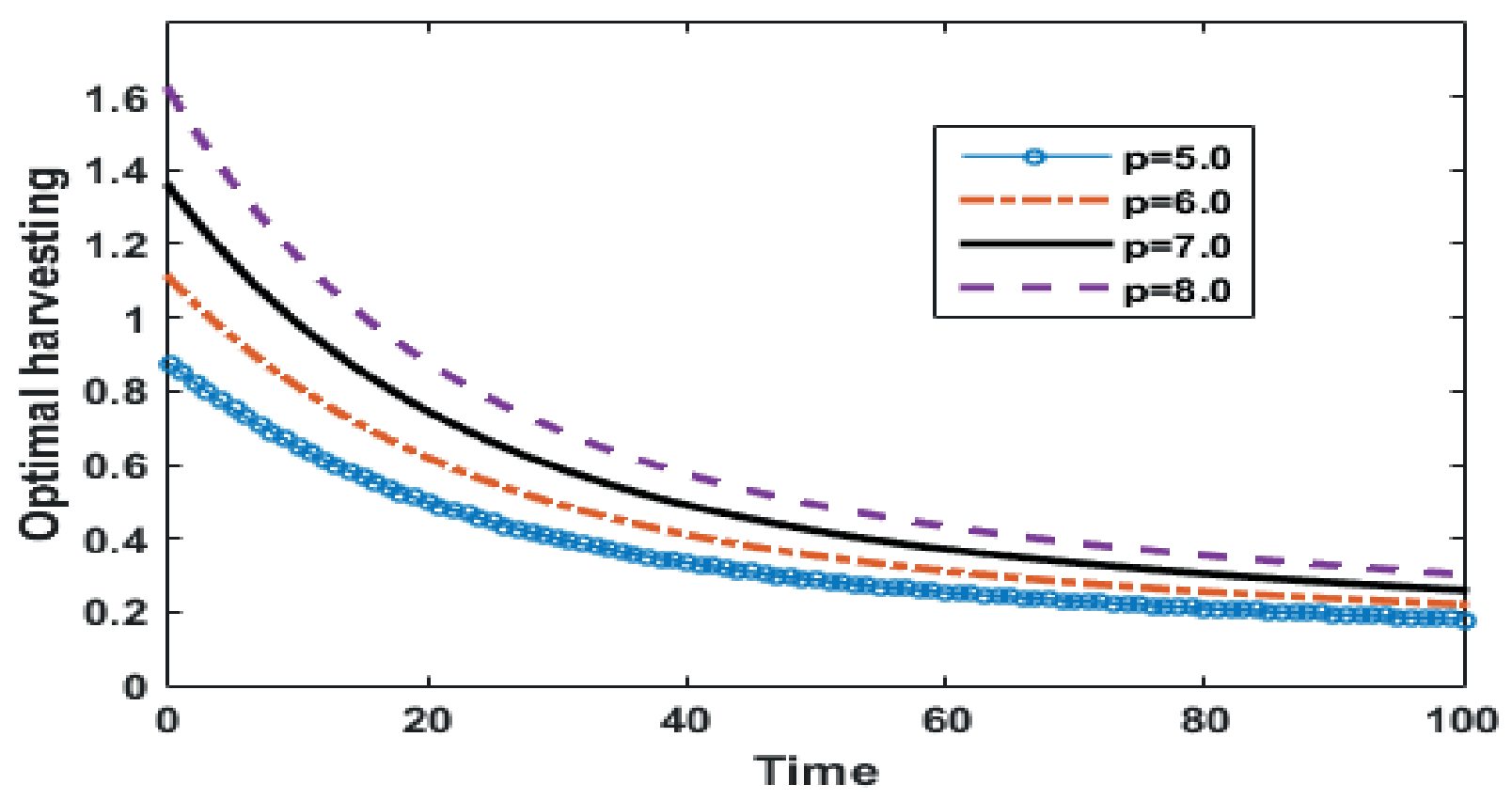

Figure 5: Optimal harvesting of Fish species with resect to selling price $(p)$.

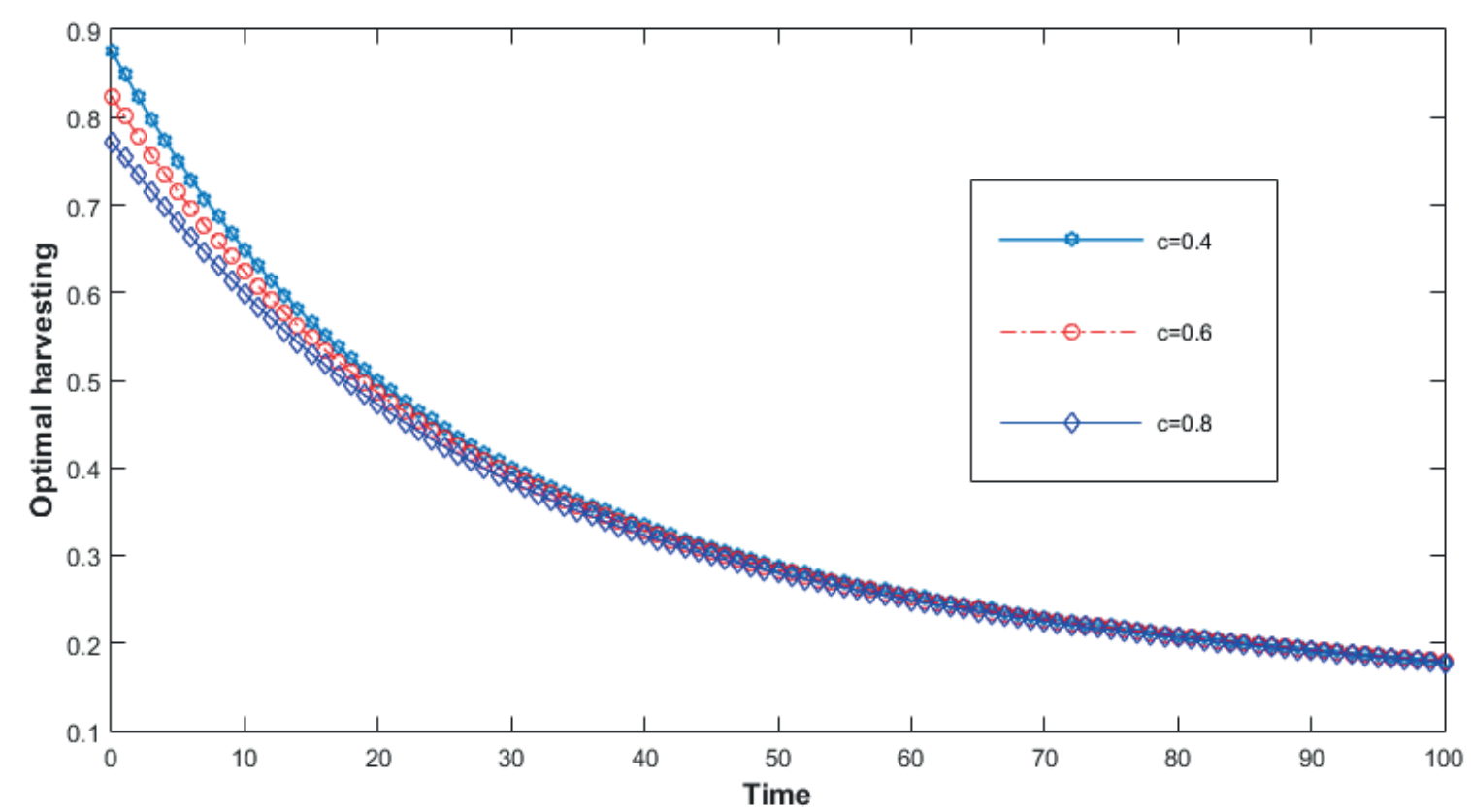

Figure 6: Optimal harvesting of Fish species with resect to harvesting cost $(c)$. 


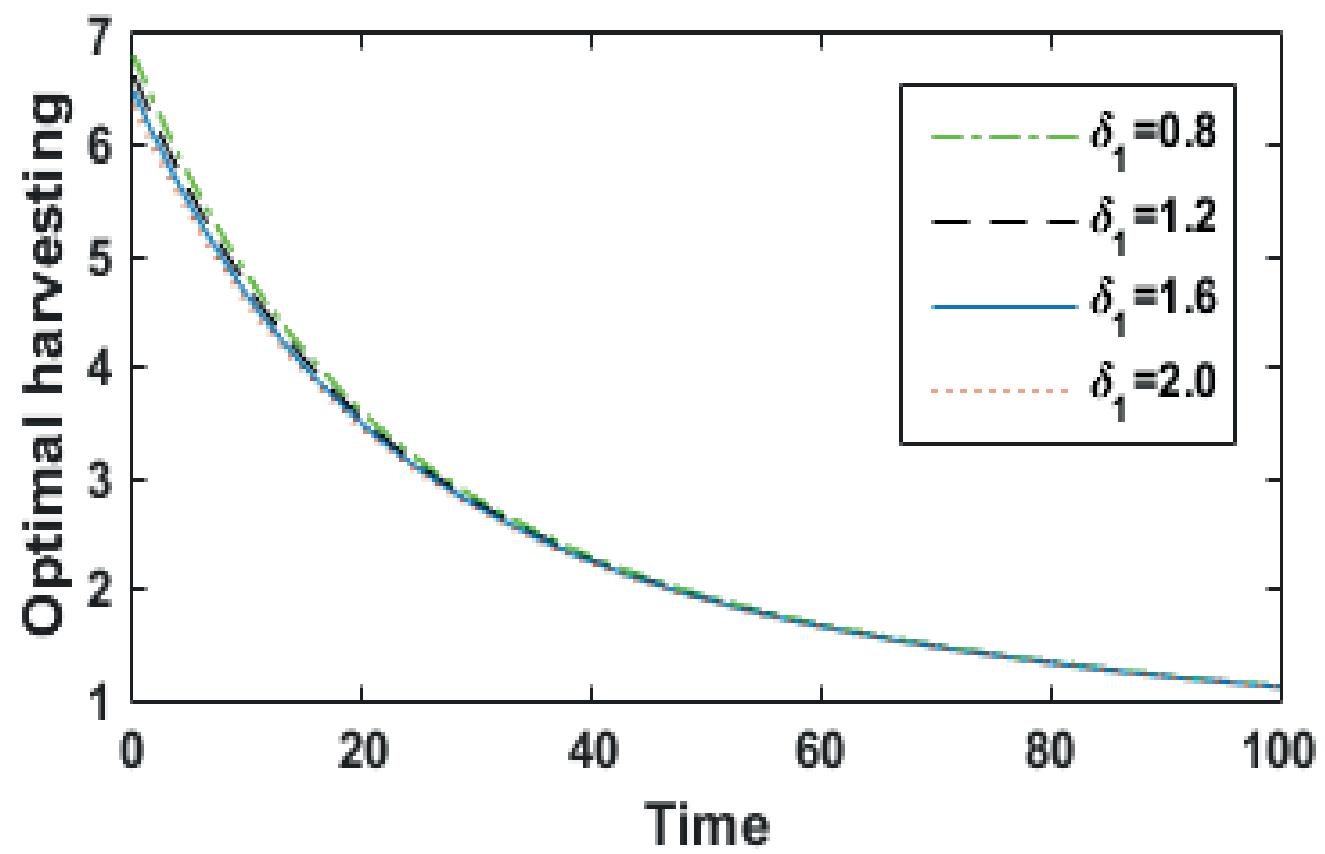

Figure 7: Optimal harvesting of Fish species with resect to annual discount rate $\left(\delta_{1}\right)$ of Fish production cost.

\section{Conclusion}

In this paper, a three species predator-prey model among the interaction of Phytoplankton, Zooplankton and Fish has been developed. Logistic growth of Phytoplankton has been considered in the absence of Zooplankton and Fish. It is assumed that Phytoplankton is consumed by Zooplankton and Fish consumes Phytoplankton as well as Zooplankton. It is considered that consumption rate of Zooplankton by Fish is greater than the consumption rate of phytoplankton by Fish. That's why two types of functional responses Holling I \& II have been used here. Then boundedness of all solutions of the system (1) has been studied. The local stability of the system has been studied around each of the possible equilibrium points. Also, global stability of the interior equilibrium point has been investigated by using Lyapunov function. The existence condition of Hopf bifurcation has been studied with respect to the half saturation constant $\alpha$. Then the optimal harvesting rate of Fish species has been determined with the help of optimal control theory.

From our numerical simulation results, it is found that the half saturation constant $(\alpha)$, the harvesting rate of Fish species $(E)$ and the consumption rate of Zooplankton by Fish $\left(\gamma_{1}\right)$ can change the system dynamics. The system loses its stability whenever $\alpha<1.18$ and 
the system remains stable if $\alpha>1.18$ (See Figure 2). It can be concluded that due to the increase of half saturation constant (increase of capability of consumption of food), the system may go into the stable state behaviour. Also, it is found that the system continues oscillatory behaviour for $0.01 \leq E \leq 0.014$, but the system becomes stable for $E>0.014$ (See Figure $3)$. So, it can be concluded that optimal harvesting of Fish may make our proposed system stable. It is observed that the system continues oscillatory behaviour for $\gamma_{1}<0.00604$, but the system continues stable steady state behaviour for $\gamma_{1} \geq 0.00604$ (See Figure 4). So, it can be concluded that the system may become stable for the higher rate of consumption of Zooplankton by Fish species. From the numerical simulation results of the optimal harvesting problem, it is observed that as the selling price $(p)$ of unit biomass of Fish (See Figure 5 ) and instantaneous annual discount rate $\left(\delta_{1}\right)$ (See Figure 7 ) increases then the optimal harvesting rate of Fish gradually increases. So, it can be concluded that the harvesting of Fish species will be increased if the selling price of Fish and the annual discount of Fish production cost increases. It is also found that the optimal harvesting rate of Fish decreases due to the increase of cost of harvesting (c) of Fish (See Figure 6). Finally, it can be said that the model can be implemented in a Fishery system. Several research works are going on Phytoplankton-Zooplankton-Fish species biological dynamics. In the near future, we shall modify this model, including some biological factors such as intra-species competition on Fish species, linear harvesting of Zooplankton species, supplying of additional food to the Fish, effects of toxicants released by Phytoplankton on Fish species etc.

\section{References}

[1] Abdllaoui A.E., Chattopadhyay J., \& Arino O. (2002) Comparisons by models of some basic mechanisms acting on the dynamics of the Zooplankton toxic Phytoplankton systems. Math.Mod.Meth.Appl.Sci. 12:1421-1451.

[2] Abrams P.A. (2000) The evolution of predator-prey interactions:theory and evidence. Annu.Rev.Ecol.Syst. 31:79-105.

[3] Birkhoff G., \& Rota G.C. (1982) Ordinray differential equations. Ginn Boston.

[4] Chakraborty K., Jana S., \& Kar T.K. (2012) Global dynamics and bifurcation in a stage structured prey-predator Fishery model with harvesting. Appl.Math.Comput. 218:92719290.

[5] Chakraborty K., Jana S., \& Kar T.K. (2012) Effort dynamics of a delay-induced preypredator system with reserve. Nonlinear Dyn. 70:1805-1829. 
[6] Chattopadhayay J., Sarkar R.R., \& Mondal S. (2002) Toxin producing plankton may act as a Biological Control for planktonic bloom-field study and Mathematical Modelling. J.Theor.Biol. 215:333-344.

[7] Chattopadhyay J., \& Pal S. (2002) Viral infection on phytoplankton-zooplankton systema mathematical model. Ecol.Model. 151:15-28.

[8] Clark C.W. (1990) Mathematical bioeconomics: The optimal management of Renewable Resources. Wiley series, New York.

[9] Das K., \& Ray S. (2008) Effect of delay on nutrient cycling in phytoplankton-zooplankton interactions in estuarine system. Ecol.Model. 215:69-76.

[10] Duinker J., \& Wefer G. (1994) Das $\mathrm{CO}_{2}$ Und Die Rolle Des Ozeans. Naturwissenschahtn. 81: $237-242$.

[11] Gakkhar S., \& Negi K. (2006) A mathematical model for viral infection in toxin producing Phytoplankton and Zooplankton system. Appl.Math.Comput. 179:301-313.

[12] Gke A., Yazar S., Sekerci Y. (2020) Delay induced nonlinear dynamics of oxygenplankton interactions. Chaos.Solit.Fract. 141:110327.

[13] Holling C.S. (1959) The characteristics of simple type of predation and parasitism. Can.Entomol. 91:385-398.

[14] Jin Y., \& Wang F.B. (2020) The dynamics of a zooplankton-fish system in aquatic habitats. Nonlinear Anal:RWA. 53: 103075.

[15] Lv Y., Pei Y., Gao S., \& Li C. (2010) Harvesting of a phytoplankton-zooplankton model. Nonlinear Anal:RWA 11:3608-3619.

[16] Levy D., Harrington, H. A., \& Van Gorder R. A. (2016) Role of seasonality on predatorprey-subsidy population dynamics. J.Theor.Biol. 396:163-181.

[17] May R.M. (1974) Stability and Complexity in Model Ecosystems (Princeton University Press, NJ).

[18] Mortoja Sk.G., Panja P., \& Mondal S.K. (2018) Dynamics of a predator-prey model with stage-structure on both species and anti-predator behavior. Inf.Med.Unlocked. 10:50-57.

[19] Murray J.D. (2002) Mathematical Biology I. An Introduction (Springer).

[20] Odum E.P. (1971) Fundamenals of Ecology, Saunders, Philadelphia. 
[21] Panja P., \& Mondal S.K. (2015) Stability analysis of coexistence of three species preypredator model. Nonlinear Dyn. 81:373-382.

[22] Panja P., Mondal S.K., \& Jana D.K. (2017) Effects of toxicants on PhytoplanktonZooplankton-Fish dynamics and harvesting. Chaos.Solit.Fract. 104:389-399.

[23] Panja P., Mondal S.K., \& Chattopadhyay J. (2017a) Dynamical Effects of Anti-predator Behaviour of Adult Prey in a Predator-Prey Model with Ratio-dependent Functional Response. Asian.J.Math.Phy. 1:19-32.

[24] Panja P., Mondal S.K., \& Chattopadhyay J. (2017b) Dynamical study in fuzzy threshold dynamics of a cholera epidemic model. Fuzzy.Inf.Eng. 9:381-401.

[25] Pontryagin L.S., Boltyanskii V.G., Gamkrelidze R.V., \& Mishchenko E.F. (1962) The mathematical theory of optimal processes. Wiley, New York.

[26] Qu Y., \& Wei J. (2007) Bifurcation analysis in a time-delay model for prey-predator growth with stage-structure. Nonlinear Dyn. 49:285-294.

[27] Raw S.N., \& Mishra P. (2019) Modeling and analysis of inhibitory effect in plankton-fish model:Application to the hypertrophic Swarzedzkie Lake in Western Poland. Nonlinear Anal: RWA. 46:465-492.

[28] Saha T., \& Bandyopadhyay M. (2009) Dynamical analysis of toxin producing Phytoplankton-Zooplankton interactions. Nonlinear Anal:RWA. 10:314-332.

[29] Samayda T. (1997) What is a bloom?, A commentary. Limnol.Oceonogr. 42:1132-1136.

[30] Sarkar R.R., \& Chattopadhyay J. (2003) The role of environmental stochasticity in a toxin Phytoplankton-non-toxic Phytoplankton Zooplankton system. Environmetrics. 14:775-792.

[31] Shi R., \& Yu J. (2017) Hopf bifurcation analysis of two Zooplankton-Phytoplankton model with two delays. Chaos.Solit.Fract. 100:62-73.

[32] Volterra V. (1926) Variazioni e fluttuazioni del numero dindividui in specie animali conviventi. Mem.Acad.Lincei.Roma. 2:31-113.

[33] Wang J., \& Jiang W. (2012) Bifurcation and chaos of a delayed predator-prey model with dormancy of predators. Nonlinear Dyn. 69:1541-1558.

[34] Zhang T., \& Wang W. (2012) Hopf bifurcation and bistability of a NutrientPhytoplankton-Zooplankton Model. Appl.Math.Model. 36:6225-6235. 
Prabir Panja and Dipak Kumar Jana/ A novel prey-predator quadratic harvesting model via optimal Control ...

[35] Zhang J.F., Wang S., \& Kong X. (2018) Effects of toxin delay on the dynamics of a phytoplankton-zooplankton model. Physica A. 505:1150-1162.

[36] Zheng W., \& Sugie J. (2019) Global asymptotic stability and equiasymptotic stability for a time-varying phytoplankton-zooplankton-fish system. Nonlinear Anal:RWA. 46:116136. 